\title{
A Kalman Filter-Based Algorithm for Measuring the Parameters of Moving Objects
}

\author{
Dimitar Dichev ${ }^{1}$, Hristofor Koev ${ }^{1}$, Totka Bakalova ${ }^{2}$, Petr Louda ${ }^{3}$ \\ ${ }^{1}$ Department of Machine and Precision Engineering, Faculty of Machine and Precision Engineering, \\ Technical University of Gabrovo, 4 Hadji Dimitar Street, 5300 Gabrovo, Bulgaria, \\ dichevd@abv.bg, koevh@abv.bg \\ ${ }^{2}$ Institute for Nanomaterials, Advanced Technologies and Innovation, Technical University of Liberec, \\ 2 Studentska Street, 46117 Liberec, Czech Republic, tbakalova@seznam.cz \\ ${ }^{3}$ Department of Material Science, Technical University of Liberec, 2 Studentska Street, \\ 46117 Liberec, Czech Republic, petr.louda@tul.cz
}

\begin{abstract}
One of the most complex problems in measuring equipment is related to the provision of the required dynamic accuracy of measuring systems determining the parameters of moving objects. The present paper views an algorithm for improving the dynamic accuracy of such measuring systems. It is based on the Kalman method. The algorithm aims to eliminate the influence of a number of interference sources, each of which is of secondary significance. However, their total effect can cause considerable distortion of the measurement signal. The algorithm model is designed for gyro-free measuring systems. It is based on one of the most widely used elements in the dynamic systems, namely the physical pendulum, due to which measuring systems of high dynamic accuracy and low cost can be developed. The presented experimental results confirm the effectiveness of the proposed algorithm with respect to the dynamic accuracy of measuring systems of this type.
\end{abstract}

Keywords: Kalman filter; dynamic error; gyro-free measuring system; roll, pitch, heel, trim.

\section{INTRODUCTION}

$\mathrm{A}$ S IT IS WELL-KNOWN $[1,2,3]$, most of the existing measuring systems are non-linear. The Nonlinear System Theory is a sophisticated and not welldeveloped theory. Therefore, the analysis done with its help is approximate. At the same time there are too many nonlinear measuring systems which are linearized by introducing a certain number of simplifying hypotheses, i.e. the measurement result is determined by means of linear mathematical models rather than by nonlinear ones.

On the other hand, the nonlinear properties of most of those measuring systems are important when forming their accuracy characteristics. The application of linear models, in some cases, leads to inadmissibly large measurement errors. In addition, the complexity and the large variety of properties of those measuring systems do not allow us to use a universal approach to their analysis and synthesis.

For example, when seeking optimal algorithms for signal processing in some measuring systems, we have to use some statistical models of the measurement signals and interference effects. Upon forming these models, the concept of linearity, stationarity and normal distribution is often used $[4,5]$. However, the above listed principles are not always applied into practice.

A possible solution to this problem is the application of adaptive algorithms which allow the measuring system to self-adjust depending on the statistical parameters of the input signal. In this way the differences between the linear model and the nonlinear nature of the system do not have a substantial impact on the formation of the measurement result.

The synthesis of measuring instruments and systems determining the parameters of moving objects, in particular instruments measuring the roll, pitch, heel and trim of a ship, can be considered in this context. These instruments must include elements modelling the basic coordinate system $[6,7]$. This enables us to determine the position of the moving object when it rotates around its centre of mass, as well as when it moves along with its centre of mass. There are different methods and tools for building and keeping the vertical in measurement mode $[7,8]$. However, gyroscopic systems are the most frequently used due to their stability against the inertial effects caused by the motion of the object (the ship).

On the other hand, the measuring instruments built on the basis of gyro-verticals are distinguished for a number of disadvantages such as a sophisticated design, less reliability under extreme conditions, requirement of special systems ensuring the gyro-vertical operation; large sizes, high prices, etc. $[7,8]$, thus limiting, to a great extent, their application.

A complete solution to those problems can be found by means of the concept proposed in [9], where unlike the existing measuring methods and tools that use vertical stabilization in the inertial space this approach is based on methods eliminating the dynamic error in real time. The proposed concept for modelling measuring instruments and systems of this type overcomes, to a great degree, the above listed disadvantages. However, due to the deviations of the measuring system model, caused by its linearization, as well as by the presence of interference sources of random characteristics and additional secondary processes of unpredictable behaviour, additional errors can occur, thus considerably reducing the measurement accuracy.

Therefore, the introduction of an appropriate adaptive algorithm developed on the minimum dynamic error criterion in the metrological chain of this type of measuring systems is an important condition for ensuring high accuracy. 


\section{BLOCK DIAGRAM OF THE MEASURING SYSTEM}

The automatic control systems and the measuring systems differ in structure since the latter have a primary transformer at its input, whose input signal is inaccessible for both immediate measurement and correction. Measuring system, as a whole, does not make the provision of output-input feedbacks in their metrological circuits possible. Therefore, it is not possible to immediately use the results from the automatic control methods in the measuring systems. However, additional measuring channels operating in parallel with the basic channels can be connected to the metrological circuits. The additional channels may possess the specific structure of correcting devices used for reducing the dynamic error. Within them the concepts of a number of methods from the automatic control theory can be effectively used.

In compliance with the basic principles of the above mentioned approach, a specific system for measuring the roll, pitch, heel and trim of a ship is developed in [9]. It overcomes the disadvantages of the existing measuring instruments since it is based, on one hand, on a very simplified mechanical module, and on the other hand, on the advanced achievements in the area of nanotechnologies, microprocessor and computer equipment.

The block diagram of the system is shown in Fig.1. It is based on a gyro-free reproduction of the vertical. The latter is developed by using a simplified design and a physical pendulum. The measuring system consists of two measuring channels operating in parallel (Fig.1.). The data obtained from the basic measuring channel contains a dynamic error mainly caused by the deviations of the physical pendulum from the ideal astronomical vertical. These fluctuations are determined by the inertial effects caused by the ship fluctuations (e.g. heave, sway, surge, roll, pitch, yaw) and the vibrations in the place where the system has been mounted.

Unlike the existing measuring methods and tools which stabilize the vertical in the inertial space so as to improve the dynamic accuracy, the proposed approach involves a method for eliminating the dynamic error in real time. The measuring procedure related to the definition of the dynamic error is performed in the additional channel (Fig.1.). The latter consists of two pairs of identical MEMS accelerometers used for measuring linear acceleration. Within the mechanical module of the system the accelerometers are positioned in such a way that an algorithm for determining the dynamic error along the two measuring coordinates (heel and trim) can be developed from their output signals. This algorithm is described in detail in [9]. The additional measuring channel operates in parallel with the basic one so as to provide a possibility for eliminating the dynamic error from the measurement result in real time.

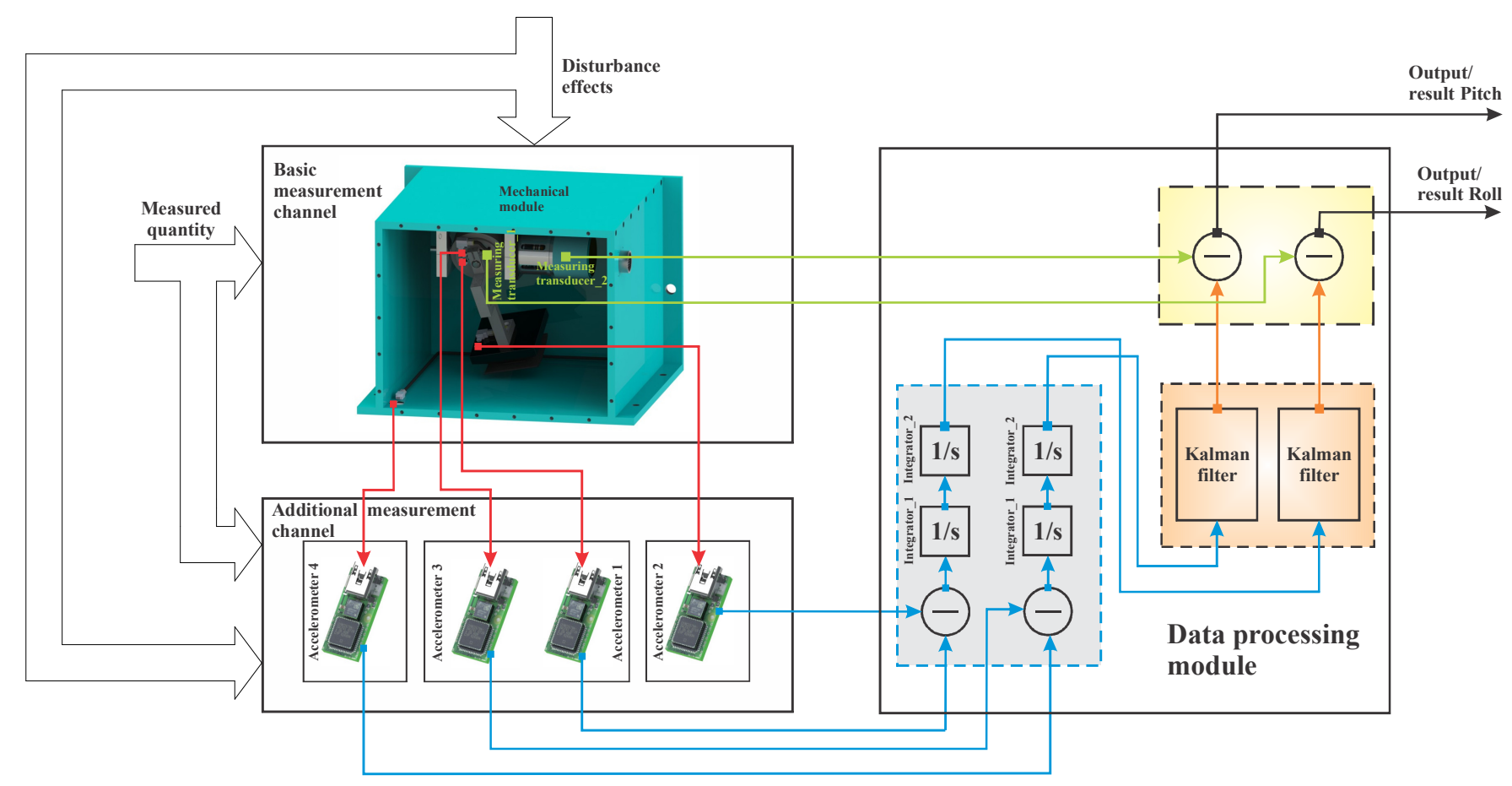

Fig.1. Block diagram of the measuring system. 


\section{STRUCTURAL MODEL OF THE ALGORITHM}

Taking into consideration the characteristics of the quantities that build the measuring environment where the measuring systems operate, it can be admitted that the most appropriate form of processing data obtained from such additional measuring channels is the Kalman algorithm. The characteristics of this algorithm fit very well into the solution of a number of problems arising in the process of accuracy optimization of the measuring instruments that define the parameters of all moving objects listed above [10]. In that case the algorithm will be developed in the context of the set objective, which is related to the improvement of the dynamic accuracy of the measuring system presented in [9]. This procedure is part of the additional channel and aims at eliminating the influence of a number of secondary disturbances on the measurement result. However, the algorithm can also be used in many other systems operating in dynamic mode in the area of metrology and automation [11-14] since the mathematical model is based on the physical pendulum, which is one of the most widely spread elements in those systems.

As it is mentioned in [9], if the dynamic error is determined only on the basis of the measuring procedure described above, the accuracy is not sufficient. The motion of the moving object initiates not only basic but also secondary disturbance processes of unpredictable behaviour for the sensitive elements of the measuring instruments in the additional channel. The mathematical model of the primary processing procedure (Fig.1.) for determining the dynamic error takes mainly the influence of the basic disturbance processes into consideration. The model remains insensitive to the secondary disturbance processes though. The magnitude and influence of the latter on the system's dynamic accuracy are different. They are determined by a number of parameters of random nature and are connected with the motion of the moving object. In some cases, however, under their influence the accumulated error can reach inadmissibly high values.

The block diagram of the Kalman filter developed for one of the two measuring channels of the system is shown in Fig.2. In this case the Kalman algorithm which measures the ship pitch and trim is viewed. However, an analogous filter with identical structure and mathematical model is connected to the metrological circuit of the second measuring channel. The block diagram can be provisionally divided into two loops operating in parallel. The first loop can be assumed as a basic one since the forecast estimate and the optimal value of the measured quantity are determined in it. The second loop operates in parallel with the first one. It aims at determining the current values of the elements from the correlation matrix of the forecast error.

To obtain the forecast estimate $\hat{\vec{x}}_{k}(-)$, it is necessary to integrate the differential equation $[15,16]$

$$
\frac{d \vec{x}(t)}{d t}=F(t) \cdot \vec{x}(t),
$$

under an initial condition $\vec{x}(0)=\vec{x}\left(t_{0}\right)$.
In (1) $\vec{x}(t)$ is the $n$ dimensional vector defining the state of the quantity whose optimal value is demanded by means of the Kalman filter developed. According to the block diagram in Fig.1. and the proposed processing procedure of the measuring system, vector $\vec{x}(t)$ is determined by the motion of the physical pendulum along the $\beta$ coordinate, which actually defines the dynamic error in this measuring channel. Error $\beta$ is explained in detail in $[9,17,18]$. By transforming equation (1) from a continuous time function into a discrete time function, the following expression is obtained for the forecast estimate [16]

$$
\hat{\vec{x}}_{k}(-)=\Phi_{k} \cdot \hat{\vec{x}}_{k-1}(+),
$$

where $\Phi_{k}$ is a transition function of the $[n \times n]$-dimensional state defining the dynamics of the system; $\hat{\vec{x}}_{k-1}(+)-$ the estimate of the vector of the state at the moment of time $t_{k-1}$.

Due to the stationary nature of the dynamic system, caused by the invariability of its basic parameters, the $\Phi_{k}$ matrix does not change in time and its elements remain constant [2].

It is necessary to define the mathematical model of the coefficient correcting the forecast estimate since the Kalman filter operates according to the forecast-correction circuit (2). Therefore, the continuous model of the linear dynamic system should be viewed. The latter could be illustrated by a vector-matrix differential equation defining the dynamics of the measuring system, and by a second equation defining the measuring process $[7,15]$

$$
\begin{gathered}
\frac{d \vec{x}}{d t}=F(t) \cdot \vec{x}(t)+G(t) \cdot \vec{w}(t), \\
\vec{z}(t)=\vec{y}(t)+\vec{v}(t)=H(t) \cdot \vec{x}(t)+\vec{v}(t),
\end{gathered}
$$

where $\vec{w}(t)$ is $r$ dimensional vector representing the signal at the system input; $\vec{y}(t)-m$ dimensional vector determining the error-free signal at the output of the measuring channel before the Kalman filter; $\vec{z}(t)-m$ dimensional vector defining the measurement result before the Kalman filter; $\vec{v}(t)-m$ dimensional vector determining the measurement error; $F(t), G(t), H(t)$ - respectively $[n x$ $n],[n \times r],[m \times n]$ matrices, which will be described later in equations (10) and (12).

The diagram in Fig.2. illustrates the used method in an algorithmic form. In this figure the error correlation matrix is denoted by $P_{k}$, the initial value of the error correlation matrix - by $P\left(t_{0}\right)$, and the matrix amplification coefficient by $K_{k}$. The error correlation matrix, whose value is restored at each time step, makes the correction of the forecast estimate according to an optimality criterion in relation to accuracy possible. In the algorithm the estimate is corrected by means of the matrix amplification coefficient $K_{k}$, whose discrete form is presented in Fig.2. 


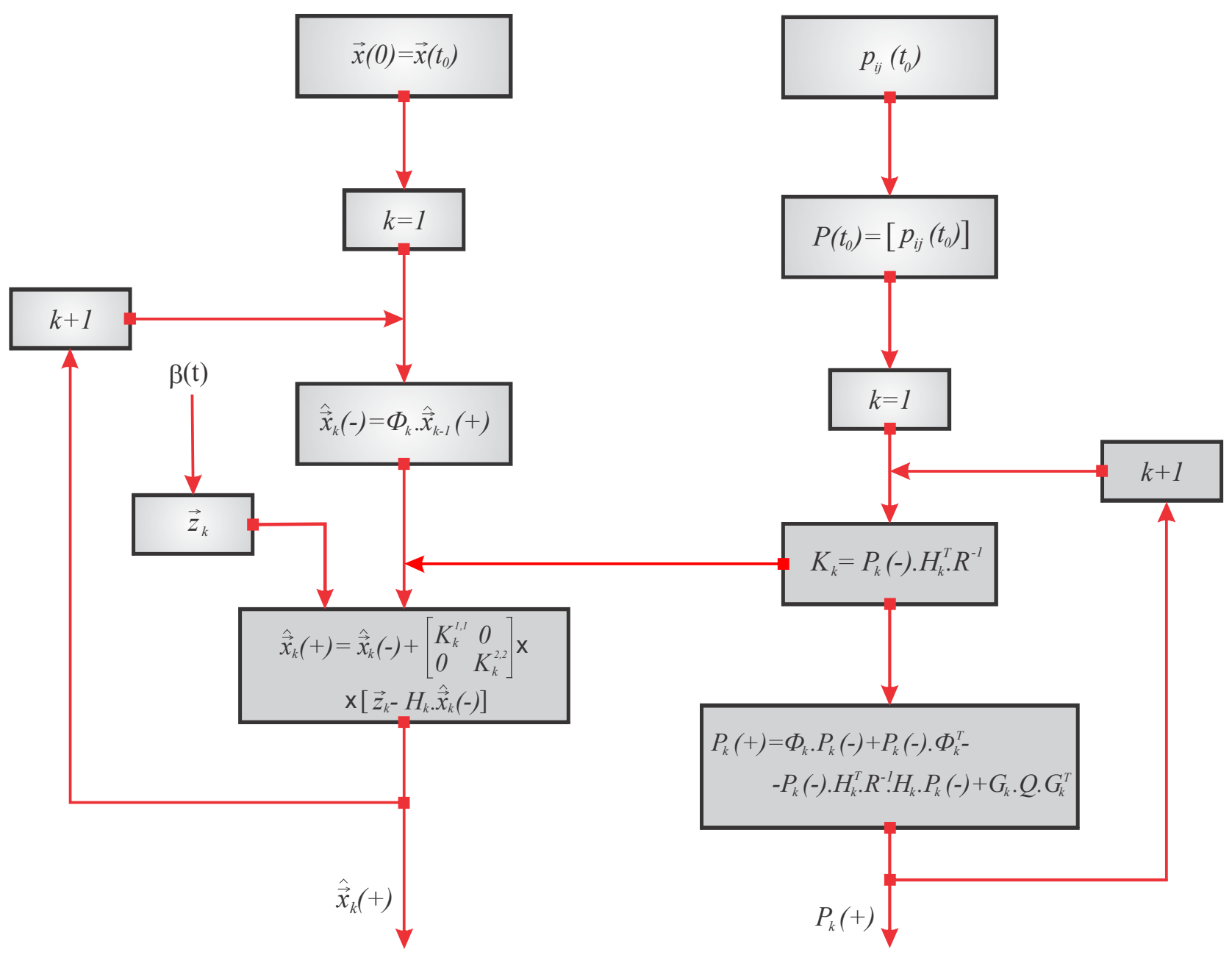

Fig.2. Block diagram of the Kalman filter.

\section{Designing OF THE ELEMENTS OF THE KALMAN FILTER}

The concept of the Kalman filter assumes that the stochastic system can be described by the models of the dynamics and measurement. In this case the dynamics model is described by the differential equation [9]

$$
\begin{array}{r}
\left(J_{x_{l}}+m_{l} \cdot l^{2}\right) \cdot \ddot{\beta}(t)+k_{\beta} \cdot \dot{\beta}(t)+m_{l} \cdot l \cdot g \cdot \beta(t)= \\
=-m_{l} \cdot l \cdot \ddot{\xi}_{0}(t)-\left(J_{x_{l}}+m_{l} \cdot l \cdot z\right) \cdot \ddot{\psi}(t),
\end{array}
$$

where $m_{l}, l, J_{x_{l}}$ are mass, design and inertia parameters of the system sensitive element; $k_{\beta}$ - damping coefficient; $\psi(t)$ pitch; $\xi_{0}(t)$ - surge; $z$ - the coordinate in the vertical direction of the suspension point of the system sensitive element with regard to the centre of gravity of the ship.

It is necessary to note that the measured quantity from the system measuring channel under consideration is $\psi(t)$. According to the metrological procedure it is measured with accuracy depending to a great extent on the accuracy related to the definition of the dynamic error $\beta(t)$. Namely the last quantity is subject to filtration in the present problem.

Equation (5) can be easily linearized in the type

$$
\begin{aligned}
& \ddot{\beta}(t)+2 \cdot h \cdot \dot{\beta}(t)+w_{0}^{2} \cdot \beta(t)= \\
& =-\frac{w_{0}^{2}}{g} \cdot \ddot{\xi}_{0}(t)-\frac{w_{0}^{2}}{g} \cdot\left(\frac{J_{x_{l}}}{m_{l} \cdot l}+z\right) \cdot \ddot{\psi}(t),
\end{aligned}
$$

where $\quad w_{0}=\sqrt{\frac{m_{l} \cdot l \cdot g}{\left(J_{x_{l}}+m_{l} \cdot l^{2}\right)}}$ - the natural frequency of the sensitive element (the physical pendulum); $2 . h=\frac{k_{\beta}}{\left(J_{x_{I}}+m_{l} \cdot l^{2}\right)} ; h$ - damping factor.

Quantities $\ddot{\xi}_{0}(t), \ddot{\psi}(t)$ in the right part of equations (5) and (6) are random time functions. The Kalman method introduces a simplifying hypothesis according to which these quantities are assumed as random functions of a white noise type $[15,16,19]$. Then equation (6) will be transformed into

$$
\ddot{\beta}(t)+2 \cdot h \cdot \dot{\beta}(t)+w_{0}^{2} \cdot \beta(t)=k_{l} \cdot w(t),
$$

where $w(t)$ - scalar white noise of intensity $Q=1$; 


$$
k_{l}=-\frac{w_{0}^{2}}{g} \cdot\left(\frac{J_{x_{1}}}{m_{l} \cdot l}+z+1\right) \text {. }
$$

The second order equation (7) can be transformed into a system of two equations of first order

$$
\begin{gathered}
\dot{\beta}(t)=\chi(t), \\
\dot{\chi}(t)=-2 \cdot h \cdot \chi(t)-w_{0}^{2} \cdot \beta(t)+k_{l} \cdot w(t) .
\end{gathered}
$$

Vector $\vec{x}(t)$ determining the state of the quantity $\beta(t)$, in compliance with (8), will be

$$
\vec{x}^{T}(t)=\left[\begin{array}{ll}
\beta & \chi
\end{array}\right] .
$$

Then matrices $F$ and $G$ will be of the type

$$
F=\Phi_{k}=\left[\begin{array}{cc}
0 & 1 \\
-w_{0}^{2} & -2 \cdot h
\end{array}\right], \quad G=G_{k}=\left[\begin{array}{l}
0 \\
k_{l}
\end{array}\right] .
$$

Within the specific problem, the equation of the measured signal $z(t)$ before the filter can be written in the following way

$$
z(t)=\beta(t)+v(t)
$$

where $v(t)$ is the measurement error which can be considered white noise of intensity $R=1$.

Then matrix $H$ from equation (4), determined by the type of the input signal entering the Kalman filter (11), will be

$$
H=H_{k}=\left[\begin{array}{ll}
1 & 0
\end{array}\right] \text {, }
$$

The diagonal matrix $P\left(t_{0}\right)$, whose elements are equal to the variances $D_{\beta}$ and $D_{\chi}=D_{\dot{\beta}}$ of the components of the vector $\vec{x}^{T}(t)=\left[\begin{array}{ll}\beta & \chi\end{array}\right]$, is written on the basis of the correlation functions of quantities $\beta(t)$ and $\dot{\beta}(t)=\chi(t)$. This is due to equations $D_{\beta}=K_{\beta}(0)$ and $D_{\dot{\beta}}=K_{\dot{\beta}}(0)$, where $K_{\beta}(0)$ and $K_{\dot{\beta}}(0)$ are the values of the correlation functions of $\beta(t)$ and $\dot{\beta}(t)=\chi(t)$ when $\tau=0$ [20].

The spectral density of the stationary solution of the linear differential equation with constant coefficients (7) can be presented in the type [20]

$$
S_{\beta}(\omega)=|W(j \omega)|^{2} \cdot S_{w}(\omega),
$$

where $W(j \omega)$ is the transfer function $W(p)$ written with the argument $p=j . \omega$.

From (7) it follows that the transfer function of the system sensitive element will be

$$
W(p)=\frac{k_{1}}{p^{2}+2 \cdot h \cdot p+w_{0}^{2}} .
$$

By replacing the arguments in (14), the following is obtained

$$
W(j \omega)=\frac{k_{1}}{-\omega^{2}+2 \cdot h \cdot j \cdot \omega+w_{0}^{2}} .
$$

Then on the basis of (13),

$$
S_{\beta}(\omega)=\frac{k_{1}^{2}}{\left(\omega^{2}-w_{0}^{2}\right)^{2}+4 \cdot h^{2} \cdot \omega^{2}} \cdot S_{w}(\omega) .
$$

Since the process $w(t)$ is white noise, then on the basis of the qualities of the latter it follows that the spectral density $S_{w}(\omega) \approx c=$ const. Then equation (16) can be transformed into the type

$$
S_{\beta}(\omega)=\frac{2 \cdot D_{\beta} \cdot \mu}{\pi} \cdot \frac{\mu^{2}+\lambda^{2}}{\left(\omega^{2}-\lambda^{2}-\mu^{2}\right)^{2}+4 \cdot \mu^{2} \cdot \omega^{2}},
$$

where $\mu=h ; \lambda=\sqrt{w_{0}^{2}-h^{2}} ; D_{\beta}=\sigma_{\beta}^{2}=\frac{\pi \cdot k_{1}}{2 \cdot h \cdot w_{0}^{2}} \cdot c$.

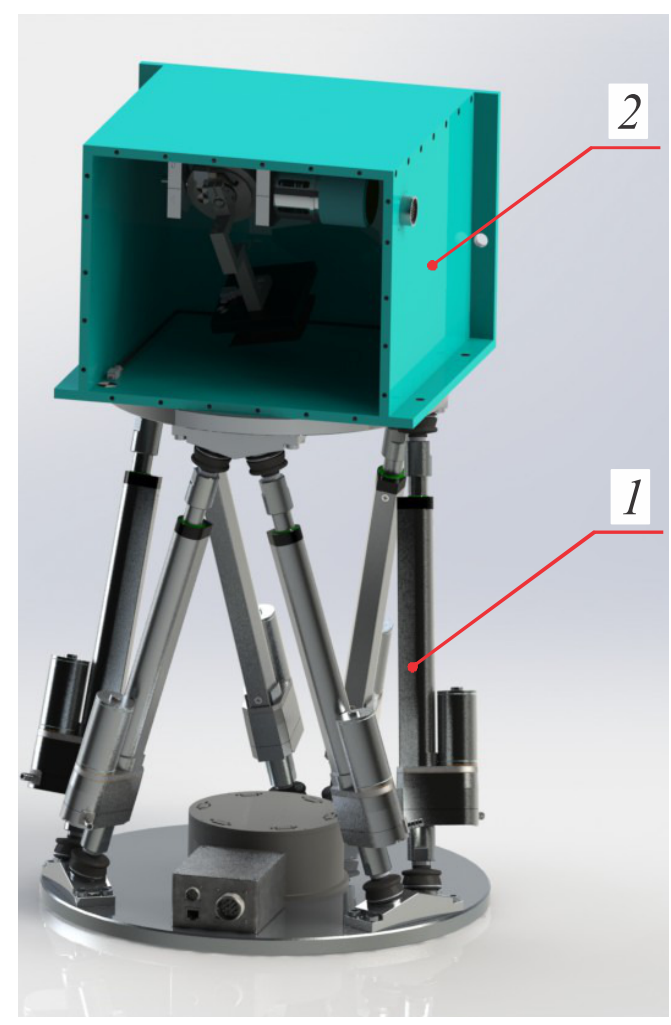

Fig.3. 3-D model of the equipment and the measuring system under study. 1 -equipment for reproducing reference motions; 2 - measuring system under study. 
From equation (17) the sought mathematical model of the correlation function $K_{\beta}(\tau)$ of the process $\beta(t)$ can be found on the basis of the common relationship

$$
\begin{gathered}
K(\tau)=2 \cdot \int_{0}^{\infty} S(\omega) \cdot \cos \omega \tau \cdot d \omega, \text { i.e. [15] } \\
K_{\beta}(\tau)=D_{\beta} \cdot e^{-\mu \cdot|\tau|} \cdot\left(\cos \lambda \cdot \tau+\frac{\mu}{\lambda} \cdot \sin \lambda \cdot|\tau|\right) .
\end{gathered}
$$

Since function $\beta(t)$ is related to $\dot{\beta}(t)$ by a differential operator, their respective correlation functions will be in the following relationship [15]

$$
K_{\dot{\beta}}\left(t_{1}, t_{2}\right)=\frac{\partial^{2} K_{\beta}\left(t_{1}, t_{2}\right)}{\partial t_{1} \cdot \partial t_{2}}=-\frac{d^{2} K_{\beta}(\tau)}{d \tau^{2}}=K_{\dot{\beta}}(\tau),
$$

where $\tau=t_{2}-t_{1}$.

It follows from (19) and (20) that

$$
K_{\dot{\beta}}(\tau)=D_{\beta} \cdot\left(\mu^{2}+\lambda^{2}\right) \cdot e^{-\mu \cdot \tau \mid} \cdot\left(\cos \lambda \tau-\frac{\mu}{\lambda} \cdot \sin \lambda|\tau|\right) .
$$

Then on the basis of (19) and (21) we can write the initial state of the correlation matrix $P\left(t_{0}\right)$ of the error, i.e.

$$
P\left(t_{0}\right)=\left[\begin{array}{cc}
D_{\beta} & 0 \\
0 & D_{\beta} \cdot\left(\mu^{2}+\lambda^{2}\right)
\end{array}\right]
$$

The expressions obtained for matrices $F, G, H, P\left(t_{0}\right)$ and the specified intensities of the white noise $Q=1, R$ at the input of the dynamic system make it possible to develop the Kalman algorithm on the basis of the block diagram in Fig.2. The algorithm improves the accuracy upon determining the function $\beta(t)$, which appears as an important condition for increasing the dynamic accuracy of the measuring system. Moreover, the numerical values of the components of the given matrices are defined by the respective design parameters of the sensitive element of the system.

\section{EXPERIMENTS AND RESULTS}

The experiments have been done in order to confirm the features and suitability of the proposed algorithm for improving the dynamic accuracy of such measuring systems. There are certain difficulties in relation to the experiments since there is not a well-developed reference base. This results in designing equipment that possesses reference properties and makes possible the solution of all types of metrological problems referring to verification, calibration, definition of dynamic characteristics and investigation of dynamic accuracy. For this purpose appropriate equipment possessing all required features has been developed. It is based on parallel mechanisms (a hexapod of six degrees of freedom). A 3-D model of the equipment and the measuring system is shown in Fig.3.



Fig.4. Results from the investigation of the dynamic accuracy.

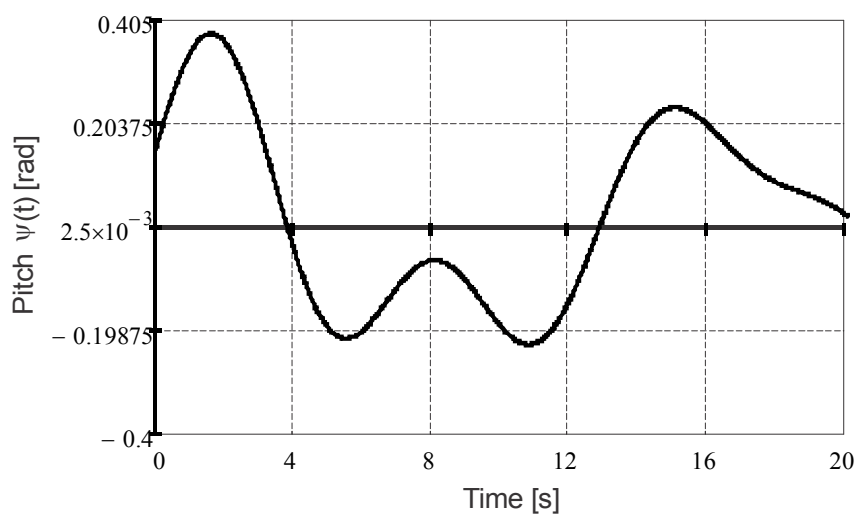

Fig.5. Motion of the operating platform along the angular coordinate $\psi$.

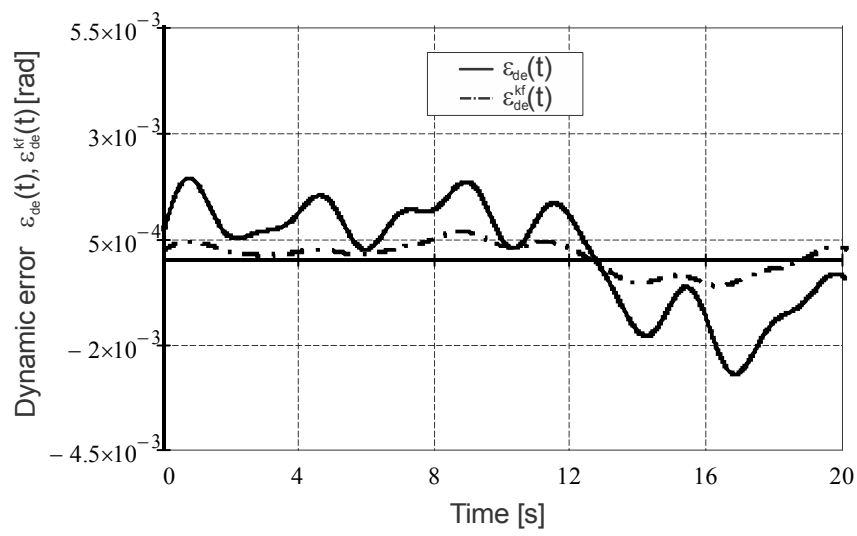

Fig.6. Results from the investigation of the dynamic accuracy.

Fig.4. and Fig.6. illustrate the effect of the operation of the proposed algorithm as regards the dynamic accuracy of the measuring system. The two figures show the dynamic errors, respectively without $\varepsilon_{d e}(t)$ (continuous line) and with $\varepsilon_{d e}^{k f}(t)$ (dotted line) an algorithm module, defined on the basis of the equations

$$
\begin{aligned}
& \varepsilon_{d e}(t)=\psi_{m r}(t)-\psi(t), \\
& \varepsilon_{d e}^{k f}(t)=\psi_{m r}^{k f}(t)-\psi(t),
\end{aligned}
$$


where $\psi_{m r}(t)$ and $\psi_{m r}^{k f}(t)$ are functions obtained as a result of the measurement of the platform motion, respectively without and with a Kalman filter; $\psi(t)$ - the function defining the motion of the operating platform in relation to the trim coordinate.

The dynamic errors shown in Fig.4. and Fig.6. are obtained as a result of the reference platform motions presented in Fig.5. and Fig.7., respectively. Fig.4. and Fig.6. show that the maximal values of the dynamic error $\varepsilon_{d e}^{k f}(t)$ vary within a range of $0.04^{\circ}-0.08^{\circ}$, whereas those of $\varepsilon_{d e}(t)$ are within a range of $0.15^{\circ}-0.25^{\circ}$. In general, we can draw the conclusion that the use of the algorithm considerably improves the stability of the measuring system with respect to its dynamic accuracy. This can be also easily identified in Fig.4. and Fig.6., where the variation of error $\varepsilon_{d e}^{k f}(t)$ decreases by approximately $50 \%$ in relation to that of error $\varepsilon_{d e}(t)$. The impact of the algorithm regarding the improvement of the dynamic accuracy is high in the presence of interference effects caused by the fluctuations of the ship in relation to the non-measured coordinates.

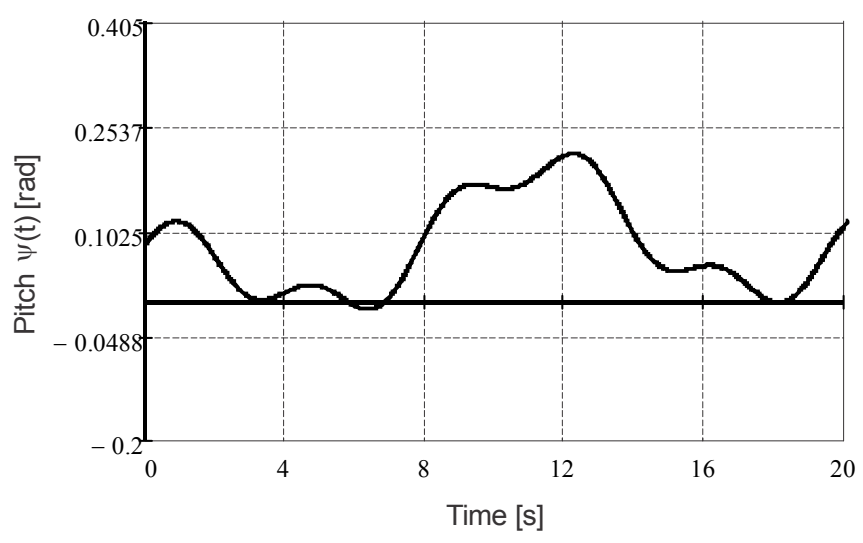

Fig.7. Motion of the operating platform along the angular coordinate $\psi$.

The experiments show that in the presence of a module processing the signal in compliance with the proposed algorithm in the measurement chain, the maximal value of the dynamic error does not exceed $0.1^{\circ}$. As an example of the effective performance of this algorithm, we can point that the maximal value of the dynamic error of the measuring system under the same measurement conditions but without using the Kalman filter is within a range of $0.2^{\circ}-0.3^{\circ}$.

\section{CONCLUSION}

The experimental results confirm the effectiveness of the proposed algorithm with respect to the dynamic accuracy of systems measuring moving objects. As a result of the operation of the algorithm the accuracy characteristics of the measuring system under conditions of dynamic influences are improved to a great extent. This can be implemented without using expensive elements and stabilization systems.

The algorithm can be successfully used for improving the dynamic accuracy in gyro-free systems measuring the parameters of moving objects. However, it can be applied in a number of other measuring instruments and systems for automation, operating in dynamic mode, since the mathematical model has been developed on the basis of a widely used element such as the physical pendulum. The algorithm is based on the Kalman method. It aims to eliminate the influence of a number of interference sources, each of which is of secondary significance. However, their total effect can cause considerable distortion of the measurement signal.

\section{ACKNOWLEDGMENT}

The research presented in this paper was supported in part by LO1201 funded by the Ministry of Education, Youth and Sports in the framework of the targeted support of the "National Programme for Sustainability I" and the OPR\&DI project Centre for Nanomaterials, Advanced Technologies and Innovation CZ.1.05/2.1.00/01.0005, as well as by Project T02-119/01. 2015 "Research and development of new methods and technologies for geometric parameters measurements of large scale details and systems", National Science Fund - Bulgaria.

\section{REFERENCES}

[1] Nazarov, N.G. (2002). Metrology: Basic Concepts and Mathematical Models. Moscow: Vishshaya Shkola.

[2] Stepetov, A.G. (2006). Theory, Calculation and Design of Measuring Instruments. Moscow: Standartinform.

[3] Pronkin, N.S. (2003). Basics of Metrology for Dynamic Measurements. Moskow: Logos.

[4] Granovskij, V.A. (1984). Dynamic Measurements. St. Petersburg: Energoatomizdat.

[5] Ventcel, E.S., Ovcharov, L.A. (2007). Random Processes Theory and Its Engineering Application. Moscow: Vishshaya Shkola.

[6] Dichev, D., Golemanova, S. (2010). Characteristics of the results upon measuring signals representing random processes. In Metrology and Metrology Assurance : XX National Scientific Symposium, 10-14 September 2010, Sazopol, Bulgaria, 51-57.

[7] Sveshnikov, A.A., Rivkin, S.S. (1974). Probability Methods in the Applied Gyroscope Theory. Moscow: Nauka.

[8] Danilov, A.T. (2001). A gyroscopic measuring system for parameters of moving objects. Problems of Special Machinebuilding Magazine, 34 (4), 178-181.

[9] Dichev, D., Koev, H., Louda, P. (2014). A measuring system with an additional channel for eliminating the dynamic error. Journal of Theoretical and Applied Mechanics, 44 (1), 3-20.

[10] Zhu, R., Sun, D., Zhou, Z., Wang, D. (2007). A linear fusion algorithm for attitude determination using low cost MEMS-based sensors. Measurement, 40 (3), 322328.

[11] Garcia, E., Hausotte, T. (2013). The parallel Bayesian toolbox for high-performance Bayesian filtering in metrology. Measurement Science Review, 13 (6), 315321. 
[12] Venkatesh, K. Arun, Mathivanan, N. (2013). CAN network based longitudinal velocity measurement using accelerometer and GPS receiver for automobiles. Measurement Science Review, 13 (3), 115-121.

[13] Sotirov, B., Tonev, D. (2013). Measurement uncertainty of simple effective diameter using measuring wires. In Materials Science, Hydro- and Aerodynamics and National Security: Third National Conference with International Participation, 24-25 October 2013. Sofia, Bulgaria: Bulgarian Academy of Sciences, 74-80.

[14] Ormandzhiev, K., Ivanov, P. (2003). Control optimization of parallel working water turbines fed by means of common pressure pipeline. In Heat Transfer, Fluid Mechanics and Thermodynamics: 2nd International Conference, 23-26 June 2003, Victoria Falls, Zambia, 56-63.

[15] Rivkin, S.S. (1974). Kalman Method for Optimal Filtration and Its Application in Navigation Systems. St. Petersburg: Sudostroene.
[16] Welch, G., Bishop, G. (2001). An Introduction to the Kalman Filter. University of North Carolina at Chapel Hill, Department of Computer Science.

[17] Dichev, D., Koev, H., Bakalova, T., Louda, P. (2014). A gyro-free system for measuring the parameters of moving objects. Measurement Science Review, 14 (5), 263-269.

[18] Dichev, D., Koev, H., Bakalova, T., Louda, P. (2014). A model of the dynamic error as a measurement result of instruments defining the parameters of moving objects. Measurement Science Review, 14 (4), 183189.

[19] Grant, P.M., Cowan, C.F., Adams, P.F. (1985). Adaptive Filters. Prentice-Hall.

[20] Ventcel, E.S., Ovcharov, L.A. (2007). Random Processes Theory and Its Engineering Application. Moscow: Vishshaya Shkola. 